\title{
Evaluación de un sistema de refuerzo estructural para un edificio: Un caso de estudio
}

\author{
M. Guzmán ${ }^{1,2}$, N. Maldonado ${ }^{1,2}$, G. Castro ${ }^{1}$, D. Buss ${ }^{1,2}$ \\ ${ }^{1}$ Universidad Tecnológica Nacional, Facultad Regional Mendoza, Coronel Rodríguez 273 Ciudad Mendoza Argentina, CP \\ 5500. \\ ${ }^{2}$ CeReDeTeC (Centro Regional de Desarrollos Tecnológicos para la Construcción, Sismología e Ingeniería Sísmica) Coronel \\ Rodríguez 273 Ciudad Mendoza Argentina, CP 5500.
}

\begin{abstract}
Información del artículo
DOI:

http://dx.doi.org/10.21041/ra.v1

$\underline{i 1.2}$

Artículo recibido el 1 de Junio

de 2010, revisado bajo las

políticas de publicación de la

Revista ALCONPAT y aceptado

el 01 de Septiembre de 2010.

Cualquier discusión, incluyendo

la réplica de los autores se publicará en el tercer número del año siempre y cuando la información se reciba antes del cierre del segundo número del año 2011
\end{abstract}

\section{(c) 2011 Alconpat Internacional}

Información Legal

Revista ALCONPAT, Año 1, No. 1, Enero Abril 2011, es una publicación cuatrimestral de la Asociación Latinoamericana de Control de Calidad, Patología y Recuperación de la Construcción, Internacional, A.C., Km. 6, antigua carretera a Progreso, Mérida Yucatán, C.P. 97310, Tel.5219997385893

C.P. $97310, T e l .5219997385893$,
alconpat.int@gmail.com, Página: alconpat.int@gmail.

Editor responsable: Dr. Pedro Castro Borges Reserva de derechos al uso exclusivo No.04 2013-011717330300-203, eISSN 2007-6835, ambos otorgados por el Instituto Nacional de Derecho de Autor. Responsable de la última actualización de este número, Unidad de actualización de este numero, Unidad de Informática ALCONPAT, Ing. Elizabeth Sabido Maldonado, Km. 6, antigua carretera a Progreso, Mérida Yucatán, C.P. 97310 , fecha de publicación: 30 de Enero de 2011

Las opiniones expresadas por los autores no necesariamente reflejan la postura del editor. Queda totalmente prohibida la reproducción total o parcial de los contenidos e imágenes de la publicación sin previa autorización de la ALCONPAT Internacional A.C.

\section{RESUMEN}

La problemática de las edificaciones existentes y cuya construcción data con anterioridad a la década de los '70 es una realidad de la cual, inexorablemente, no se puede desestimar debido a la peligrosidad que esto implica por la imprevisión en los diseños sismorresistentes.

Toda la Provincia de Mendoza, Argentina, está ubicada en una región de elevado nivel de peligro sísmico. El Gran Mendoza ha experimentado intensidades sísmicas iguales o superiores a VI M.M. por lo menos once veces en los últimos 200 años. El caso de estudio corresponde a un edificio existente ubicado en el microcentro de la ciudad de Mendoza. El mismo fue construido en la década de los '50 y dada la necesidad de su refuncionalización, se encomendó el estudio para evaluar el estado de conservación así como también la adecuación al código sismorresistente actualmente vigente en la jurisdicción de competencia. El estudio permitió establecer la necesidad de plantear un sistema integral de refuerzo estructural debido no sólo al estado de fisuración de los muros de mampostería presentes y/o al incompleto encadenamiento, sino también a la escasez de planos sismorresistentes necesarios para satisfacer la eventual demanda impuesta por el terremoto de diseño. Se tuvo en cuenta el capítulo 8 del Código de Construcciones Sismorresistentes para la Provincia de Mendoza de 1987, donde se establecen las pautas a seguir sobre las construcciones existentes que son motivo de reformas. En la propuesta de recuperación estructural se consideró el uso de pórticos metálicos arriostrados excéntricamente, los cuales presentan importantes bondades frente a otros tipos de sistemas estructurales, tales como un bajo costo de inversión, rapidez en las tareas de rehabilitación y mano de obra no especializada.

Palabras clave: peligrosidad sísmica; refuerzo estructural; diseño estructural; pórticos metálicos.

\section{ABSTRACT}

The problematic of buildings constructed before the decade of the '70 must not be overlooked due to the latent risks from the lack of foresight regarding the earthquake resistant construction designs. The Province of Mendoza, Argentina, is located in a region of the highest level of seismic risk. The Great Mendoza has undergone seismic intensities of VI M.M and above at least eleven times in the last 200 years. This case study corresponds to an existing building in downtown of Mendoza, Argentina and built in the decade of the '50. Due to the need of its rehabilitation, this study was entrusted to evaluate the state of conservation as well to adequate it to the seismic code in force. The study established the need to include an integral system of structural reinforcement in view of the the cracking of masonry walls, the incomplete tie-reinforcement and in order to satisfy the demand imposed by the earthquake design. Chapter 8 of the 1987 Code of Earthquake Resistant Constructions for the Province of Mendoza was considered for the reforms on existing buildings. The use of steel frame braced eccentrically was considered in the proposal of structural rehabilitation, which presents advantages compared to other types of structural systems, such as a low cost, speed and unskilled workmanship.

Key words: seismic hazard, structural reinforcement, structural design, metal frames 


\section{INTRODUCCION}

La Provincia de Mendoza, Argentina, tiene el núcleo urbano expuesto al mayor riesgo sísmico del país, en la zona denominada Gran Mendoza (INPRES, 1986). Esta situación ha generado un importante trabajo de actualización de los códigos de construcciones sismorresistentes. Respecto a las construcciones existentes sobre las que se pretende ampliar, reformar y/o refuncionalizar, el Código de Construcciones Sismorresistentes para la Provincia de Mendoza del año 1987 (Consejo Profesional de Ingenieros, 1994) esboza los criterios a aplicar.

En el caso particular del edificio en estudio, el mismo fue construido antes de la entrada en vigencia del primer Código de Construcciones Antisísmicas para la Provincia de Mendoza del año 1970 (Gobierno de Mendoza, 1970), lo cual implicó la necesidad de evaluar el grado de vulnerabilidad al que se encontraría expuesto ante una eventual demanda sísmica. De esta evaluación surgen las medidas correctivas necesarias a los efectos de conseguir, refuerzo estructural mediante, reestablecer niveles de seguridad acorde a lo establecido en el código actual.

\section{METODOLOGIA DE TRABAJO}

En primer lugar se procede a la búsqueda y recopilación de toda información que pudiere establecer: fecha de construcción, calidad y tipo de materiales, detalles generales y particulares de los elementos estructurales, etc. Posteriormente se lleva a cabo un relevamiento técnico de la construcción, determinando dimensiones, estado de conservación, tipo de materiales y sistema sismorresistente prevaleciente. El relevamiento se apoya en sondeos ejecutados en diversos sectores y sobre distintos elementos. Por último en gabinete con la información recabada y con las necesidades de refuncionalización expuestas por el comitente (reformas, uso o destino pretendido) se procede a la evaluación de las condiciones actuales de la construcción y de la necesidad de medidas correctivas, proponiendo, de ser necesario el sistema de refuerzo más conveniente.

\section{DESCRIPCION DEL EDIFICIO}

\subsection{Información general}

No se encontró documentación que permitiera establecer información acerca del edificio. Sólo se pudo considerar a través del aspecto arquitectónico, que la construcción data aproximadamente de la década de los '50. Figura 1.
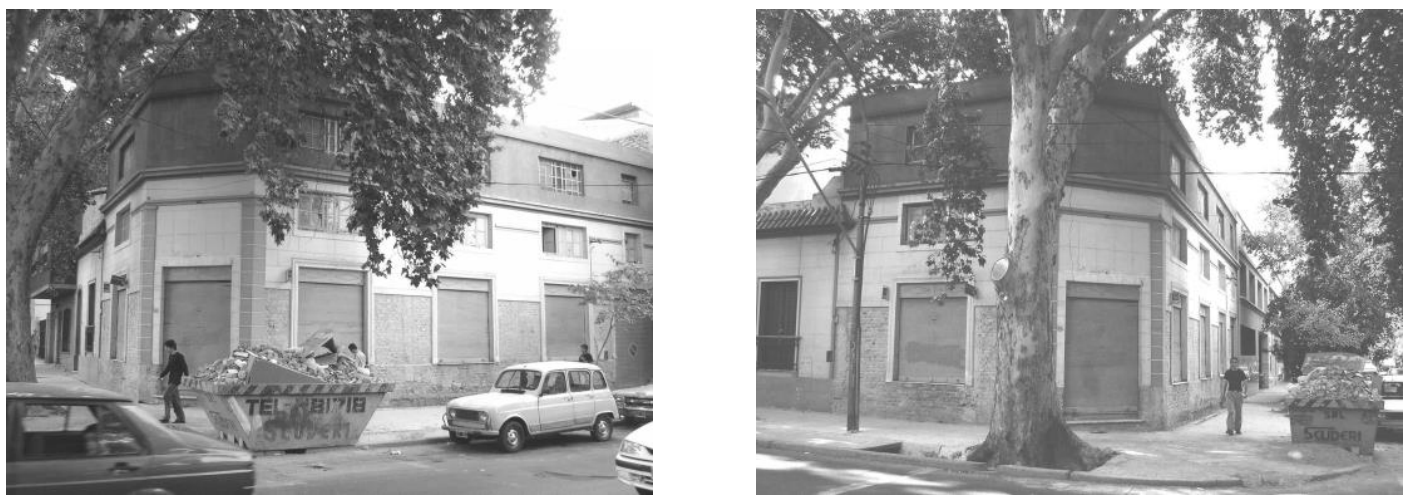

Figura 1. Fachada del edificio 


\subsection{Relevamiento}

El edificio presenta dos niveles, de dimensiones 7.60x 17.30m medidas en planta, con una altura total de aproximadamente $8.00 \mathrm{~m}$. Los cerramientos exteriores están constituidos por muros de ladrillo cerámico macizo (ladrillón) de espesor $0.28 \mathrm{~m}$. Se observa en estos muros que en algunos casos carecen de encadenado completo debido a la ausencia en uno de sus bordes del encadenado vertical (columna). Asimismo se observa el mal estado de conservación de algunas columnas producto de un avanzado proceso de corrosión del acero. Figura 2.
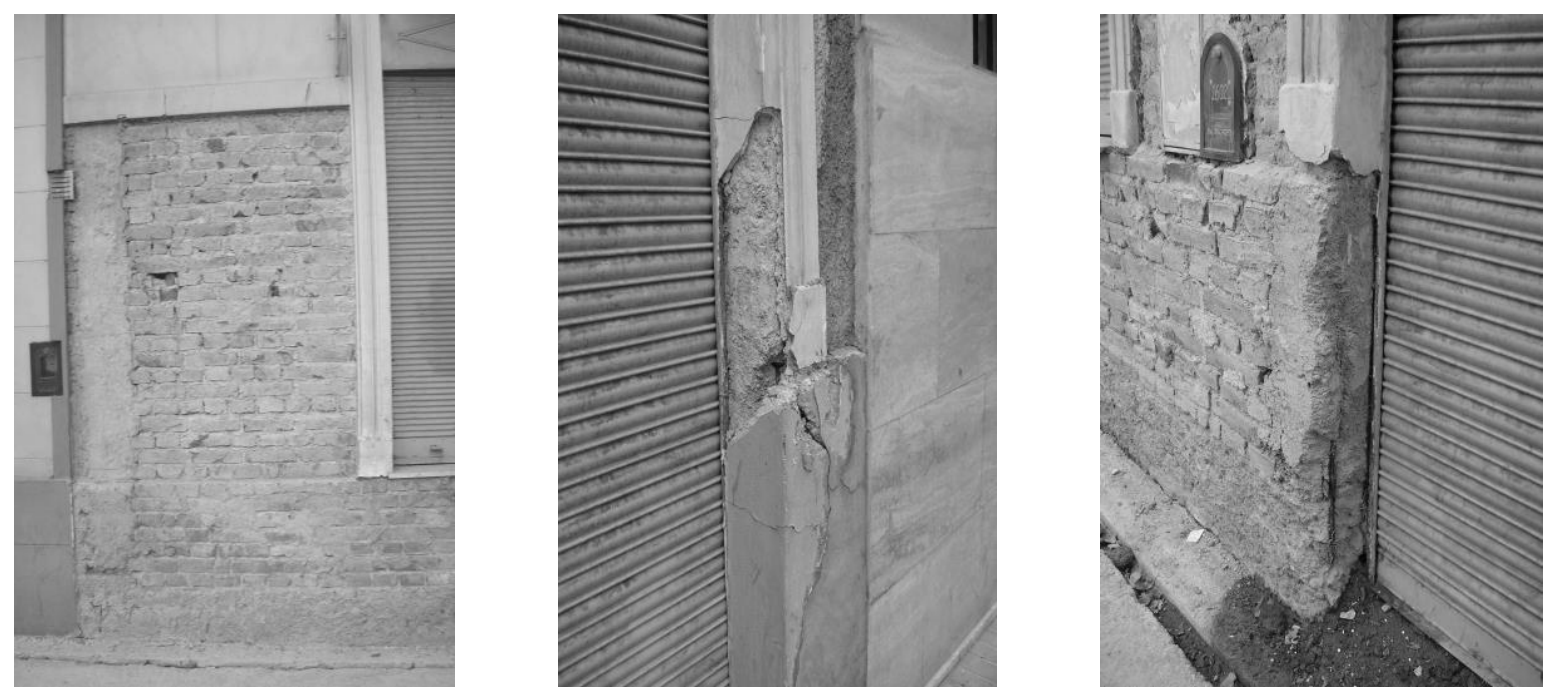

Figura 2. Falta de columna de borde - Estado de conservación de columnas de borde

El entrepiso está constituido por losas de hormigón armado de $0.13 \mathrm{~m}$ de espesor, apoyando a la mitad de su ancho en una viga de hormigón armado. Dicha viga a su vez descansa sobre cuatro columnas de hormigón armado. Figura 3
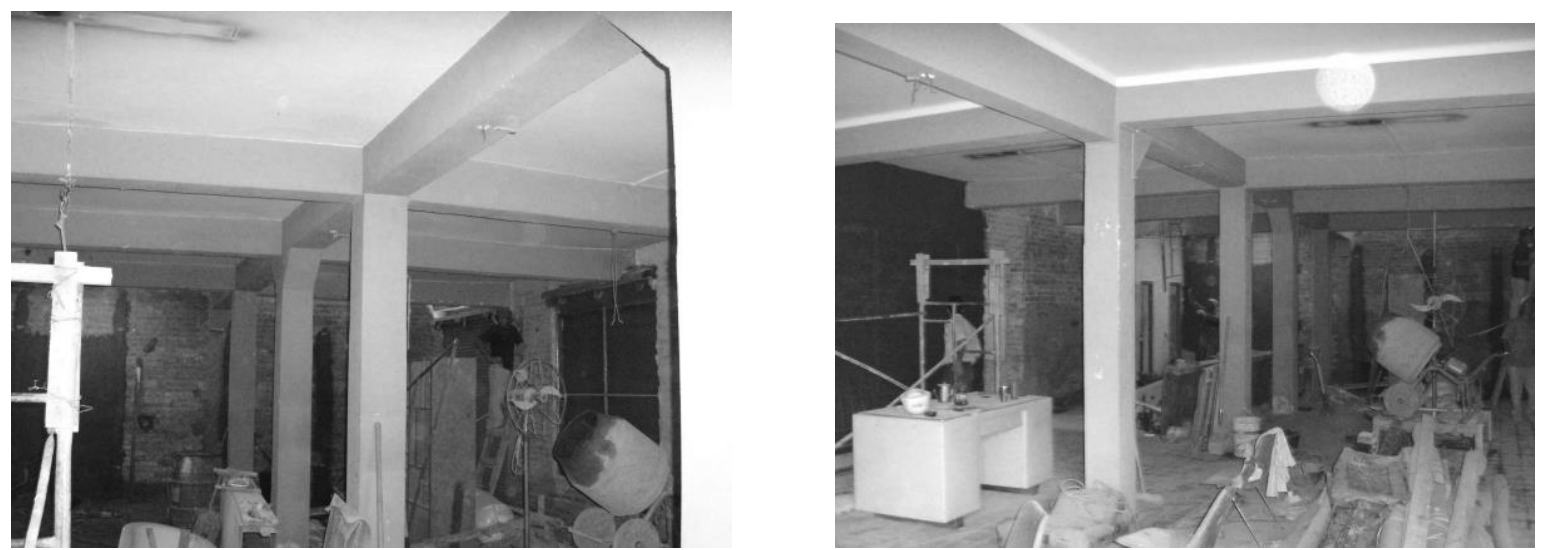

Figura 3. Losas, vigas y columnas de hormigón armado de planta baja

La cubierta de planta alta está constituida por correas de pinotea sobre la que descansan cañas, y sobre estas una capa de barro y nylon con funciones de barrera térmica e hidráulica. A la mitad de su luz las correas apoyan sobre una viga metálica constituida por dos perfiles $\mathrm{C}$ de $200 \mathrm{~mm}$ de altura unidos entre si. Esta viga metálica se apoya sobre dos columnas de hormigón armado ubicadas en el interior de la planta alta. Figura 4. 

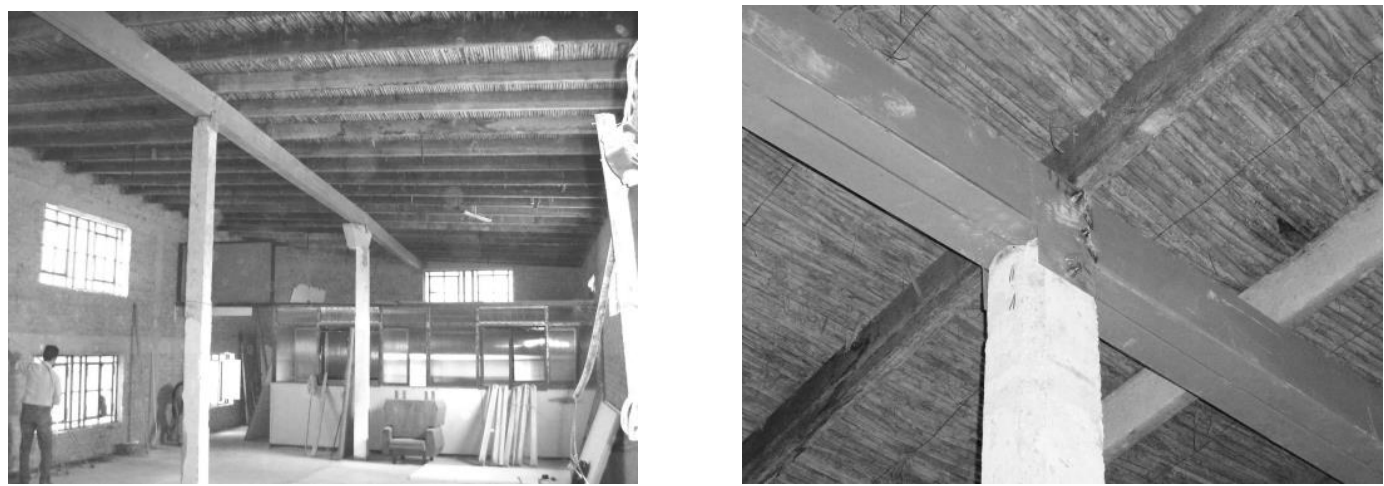

Figura 4. Columnas de hormigón armado de planta alta - Elementos de cubierta de planta alta

En lo que respecta al sistema sismorresistente de planta baja, el mismo está constituido por muros exteriores vinculados entre sí a través de un diafragma rígido (losas). A la menor dimensión de la planta (ancho) se la denomina dirección $\mathrm{x}$-x, mientras que a la mayor dimensión (largo) dirección $y-y$. En la dirección $x-x$ se encuentran dos muros ubicados en los extremos opuestos sobre el perímetro de la construcción. Estos muros presentan un encadenamiento completo, pero se observa en uno de ellos una marcada fisuración diagonal que podría deberse a la acción de esfuerzos horizontales. En la dirección y-y, y sobre uno de los lados de la construcción se encuentra un muro de mampostería con encadenado completo. Figura 5

El sistema de fundación de los muros está constituido por una base corrida de hormigón ciclópeo de $0.40 \mathrm{~m}$ de ancho con una cota de fundación de $-0.80 \mathrm{~m}$. Las columnas ubicadas en el interior de la construcción presentan bases aisladas de $0.90 \times 0.90 \mathrm{~m}$ con una cota de fundación de $-1.10 \mathrm{~m}$. Figura 6.

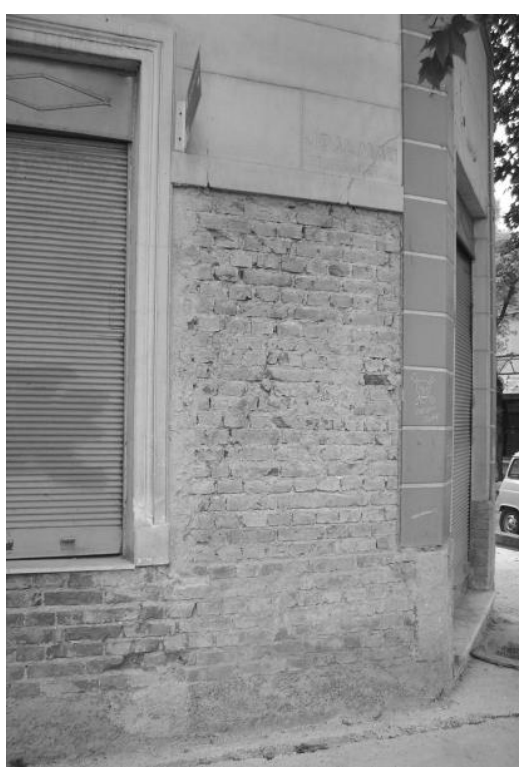

Figura 5. Muro fisurado
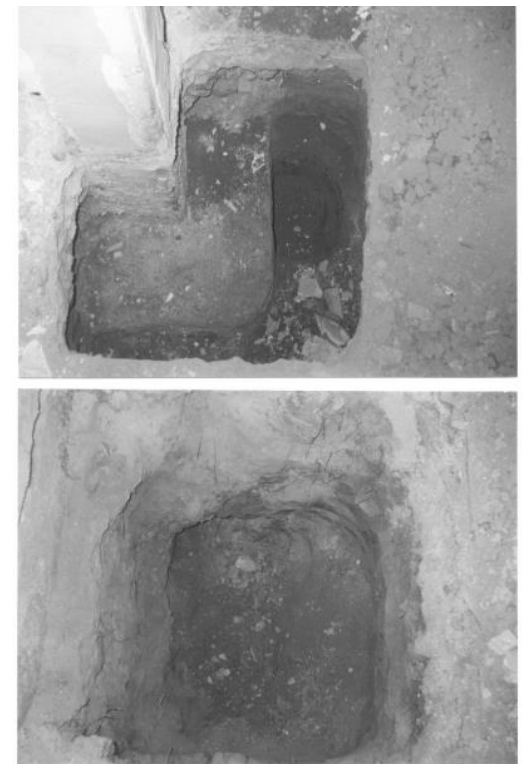

Figura 6. Fundación de columna 


\section{DIAGNÓSTICO DE LA OBRA PRIMITIVA}

\subsection{Evaluación en gabinete}

A partir de los datos aportados por el relevamiento en lo que se refiere a la época de la construcción, a su estado general de conservación, a la calidad de los materiales utilizados y a la presencia de planos estructurales sismorresistentes, se permite inferir lo siguiente:

- Los elementos considerados estructuralmente sismorresistentes dada su cantidad como calidad no alcanzan a ser suficientes como para satisfacer la demanda impuesta por el terremoto de diseño establecido en el CCSR/87 (Consejo Profesional de Ingenieros, 1994) actualmente en vigencia. Además se carece de un sistema completo de resistencia torsional.

- La seguridad actual de la obra primitiva, capítulo 8 del CCSR/87 (Consejo Profesional de Ingenieros, 1994), se estima como S4 es decir seguridad insuficiente.

- Se debe evaluar a partir de las dimensiones de losas, vigas y columnas, así como también de la sección y calidad del acero, y resistencia del hormigón utilizado, la real capacidad de estos elementos frente a la acción de cargas gravitatorias.

- Se debe llevar a cabo un estudio geotécnico del suelo a fin de obtener parámetros que permitan verificar las fundaciones existentes así como también diseñar el sistema de fundación del refuerzo propuesto.

Por lo señalado se concluye en que se deben adoptar medidas correctivas a fin de reestablecer la seguridad del edificio de acuerdo a las exigencias del CCSR/87 (Consejo Profesional de Ingenieros, 1994), teniendo presente los cambios de destinos o usos de la construcción propuestos por el comitente.

\section{PROGRAMA DE REFUERZO ESTRUCTURAL}

\subsection{Introducción}

En base al diagnóstico realizado, surge la necesidad de ejecutar sobre el edificio un programa integral de refuerzo estructural capaz de restablecer la seguridad, y de garantizar niveles de desempeño que se ajusten a los distintos estados límites (operacional-rango elástico, próximo al colapso-rango plástico).

\subsection{Tipología estructural adoptada}

Surge la necesidad de adoptar un sistema de refuerzo estructural tal que satisfaga distintos aspectos tales como:

- Comportamiento Estructural: rigidez, resistencia y ductilidad

- Economía del Sistema: costo del sistema de reforzamiento vs. Costo del inmueble

- Materialización: materiales del mercado local y mano de obra no especializada

De la evaluación de los aspectos mencionados, se concluye en adoptar como refuerzo estructural a un sistema compuesto por Pórticos de acero sismorresistentes arriostrados excéntricamente. De acuerdo a las investigaciones abordadas principalmente por Popov (Popov et al., 1989) y posteriores ensayos experimentales, indicaron que en estas estructuras se presentan ventajas excelentes en cuanto a capacidad de disipar energía mediante deformaciones plásticas así como también en una adecuada rigidez lateral para controlar desplazamientos. Estos estudios sirvieron de sustento a la American Institute of Steel Construction (AISC, 2002) base actual del Proyecto de Reglamento INPRES-CIRSOC 103 Parte IV (INPRES-CIRSOC, 2000). El citado reglamento asigna a esta tipología estructural un factor de ductilidad $\mu=6$, destacando entonces su gran capacidad de disipación de energía, que para el caso de diseñar al enlace del sistema como del tipo corto la plastificación del mismo está controlada por los esfuerzos de corte que se presenten 
en éste. Esta elevada ductilidad hace que la demanda sísmica, en comparación con otros sistemas, sea menor, dada la posibilidad de disipar energía por deformaciones plásticas. Más allá de sus ventajas técnicas, este sistema presenta la ventaja de incorporar una masa sísmica mínima a la estructura, poder ser materializado con elementos que se consiguen en el mercado local, con mano de obra no especializada, con posibilidad de trabajo en seco y fundamentalmente con permitir una transferencia segura de acciones desde la estructura primitiva al sistema de reforzamiento, sin la cual la rehabilitación resultaría ineficiente. Figura 7.
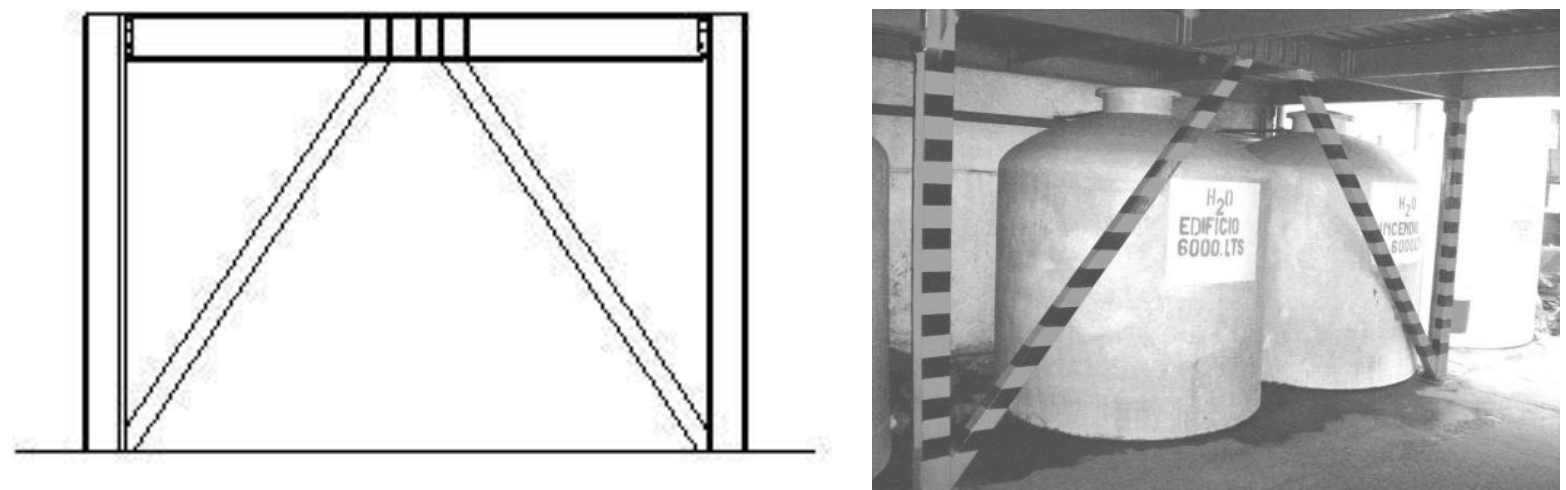

Figura 7: Esquema y ejemplo de la tipología adoptada

\subsection{Evaluación del efecto sísmico (según CCSR/87)}

El efecto sísmico se evalúa a través de un análisis lineal estático, distribuyendo la acción horizontal (Fórmula 1) en función de la rigidez de cada plano sismo resistente.

La estructura primitiva presenta planos sismorresistentes (muros), pero que podrían resultar de dudoso funcionamiento en virtud de desconocer el grado de anclaje entre encadenados, la calidad de los materiales y la posibilidad de giro de las fundaciones. Ante esto se procede a analizar distintos modelos a fin de valorar la posibilidad o no, de un funcionamiento integral de la estructura sismo resistente existente con el reforzamiento planteado, obteniendo así la envolvente de solicitaciones actuantes sobre los refuerzos.

a) Masa sísmica

Planta Alta $=850 \mathrm{kN} \quad$ Planta Baja $=790 \mathrm{kN} \quad$ Peso Total $=\mathrm{W}=1640 \mathrm{kN}$

b) Solicitaciones sísmicas (Método estático)

Coeficiente Sísmico Zonal $\rightarrow \mathrm{C}_{\mathrm{o}}=0.30$ Elevado riesgo sísmico (ciudad de Mza.)

Coeficiente de Destino $\rightarrow \gamma_{\mathrm{d}}=1.00$ Construcción según su destino: Grupo B

Coeficiente de Vinculación $\quad \rightarrow \quad \gamma_{\mathrm{vi}}=1.00$ Estructura con buena vinculación interna

Coeficiente de Ductilidad $\rightarrow \gamma_{\mathrm{du}}=0.85$ Estructura muy dúctil

Influencia del Terreno $\quad \rightarrow \quad \mathrm{S}_{\max }=1.00$ Terreno tipo 2: terrenos medios

Corte Basal

$\rightarrow \quad \mathrm{F}_{\mathrm{X}}=\mathrm{F}_{\mathrm{Y}}=\mathrm{C}_{\mathrm{O}} * \gamma_{\mathrm{d}} * \gamma_{\mathrm{vi}} * \gamma_{\mathrm{du}} * \mathrm{~s}_{\max } * \mathrm{~W}=418 \mathrm{kN}$

\subsection{Distribución de las solicitaciones sísmicas (distribución por rigidez)}

a) Modelo de análisis I

Este modelo incorpora a los planos sismorresistentes formados por los refuerzos y por los muros perimetrales que por sus condiciones (longitud, enmarcado, etc.) se consideran como eventuales planos sismorresistentes. Figura 8. 
Revista ALCONPAT, Volumen 1, Número 1, Enero-Abril 2011, Páginas 1 - 15

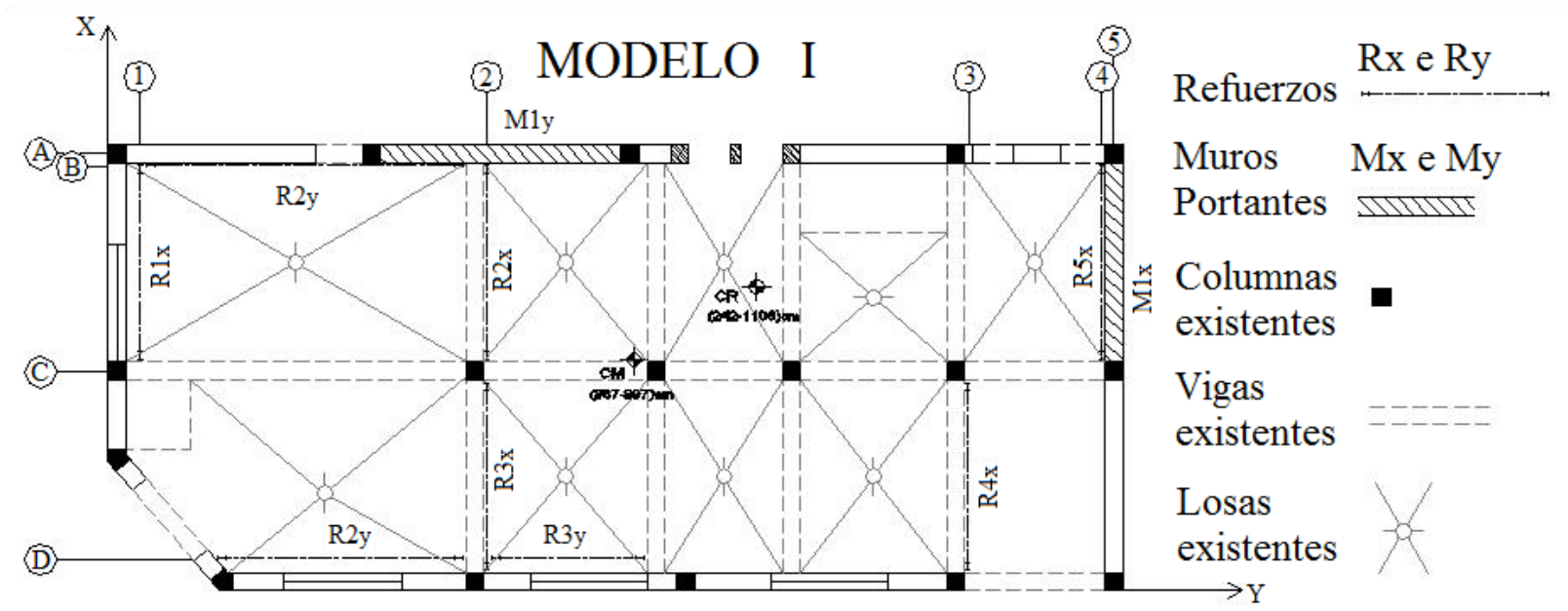

Figura 8. Planta estructura modelo de análisis I

b) Modelo de análisis II

Este modelo incorpora a los planos sismorresistentes formados por los refuerzos y solo por el muro perimetral ubicado sobre el eje $\mathrm{y}-\mathrm{y}, \mathrm{y}$ que por sus condiciones (longitud, enmarcado, etc.) se considera como eventual plano sismorresistente. Figura 9

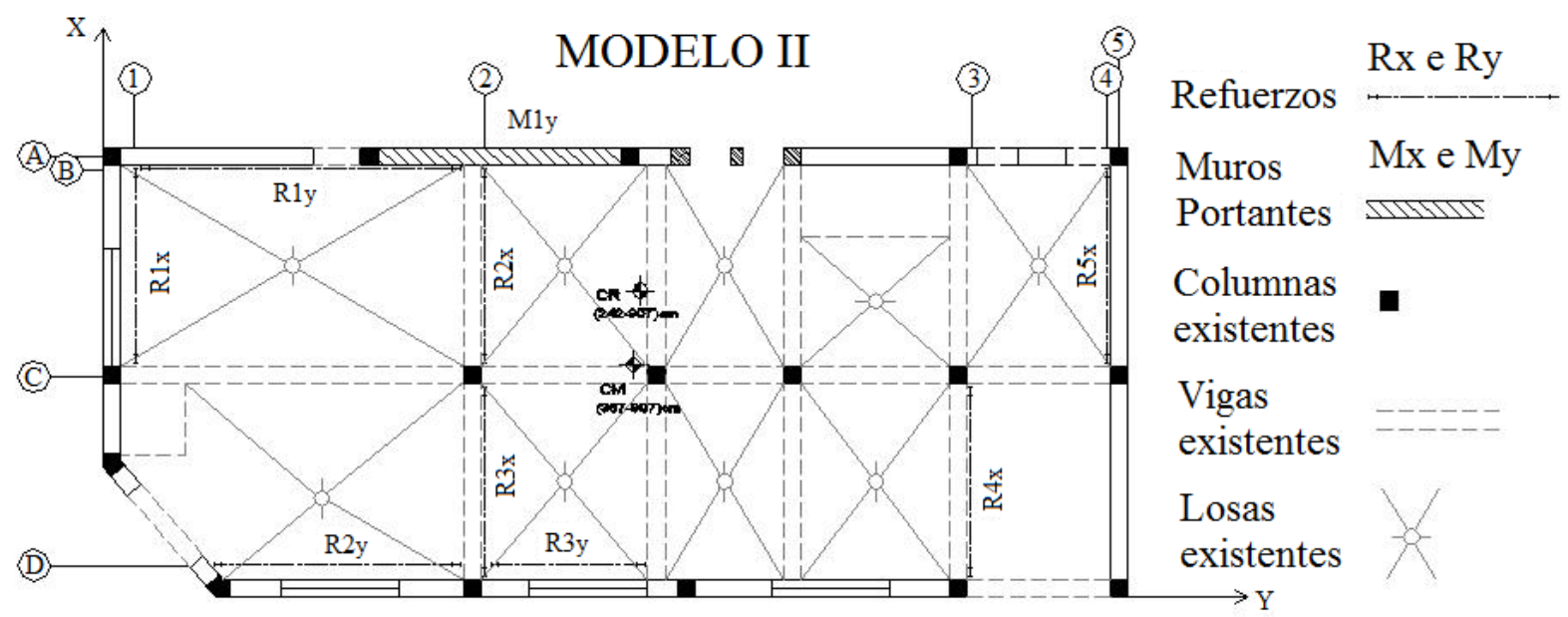

Figura 9. Planta estructura modelo de análisis II

c) Modelo de análisis III

Este modelo incorpora a los planos sismorresistentes formados por los refuerzos y solo por el muro perimetral ubicado sobre el eje x-x que por sus condiciones (longitud, enmarcado, etc.) se considera como eventual plano sismorresistente. Figura 10. 
Revista ALCONPAT, Volumen 1, Número 1, Enero-Abril 2011, Páginas 1 - 15

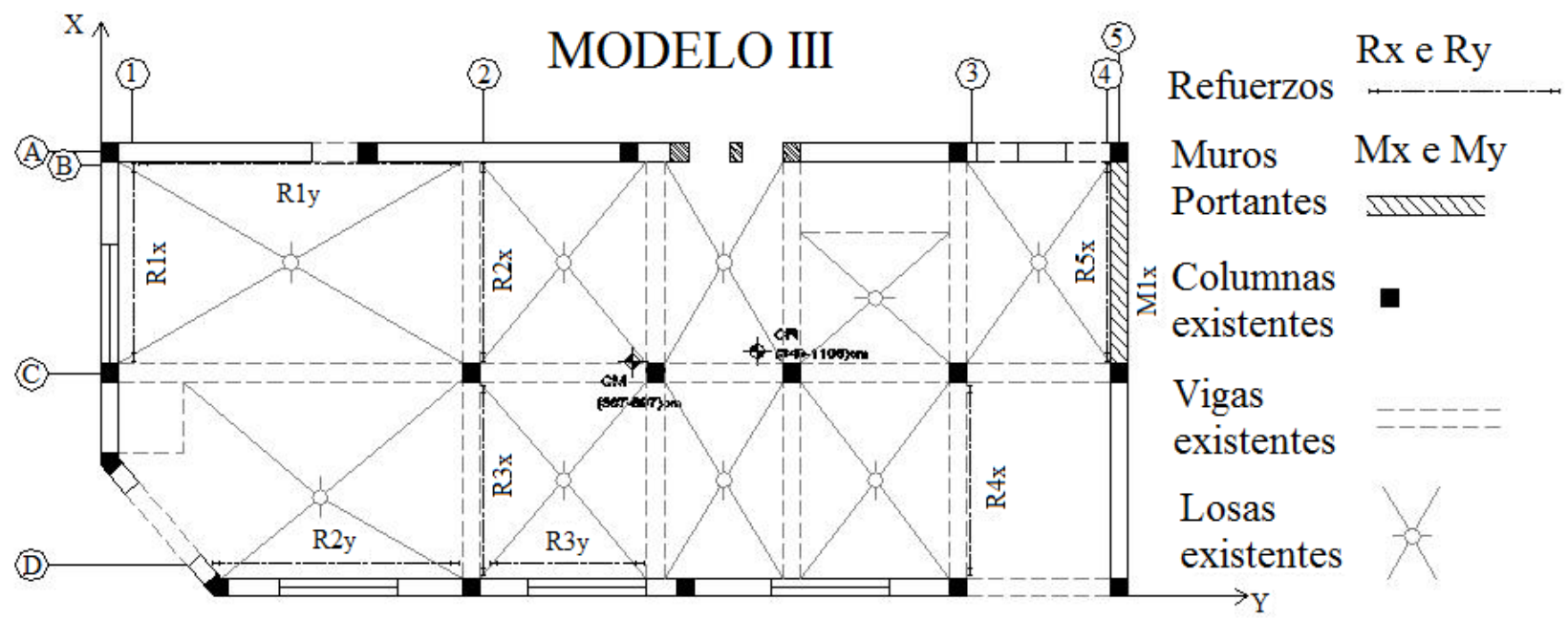

Figura 10. Planta estructura modelo de análisis III

d) Modelo de análisis IV

Este modelo incorpora solo los refuerzos como planos sismorresistentes. Figura 11

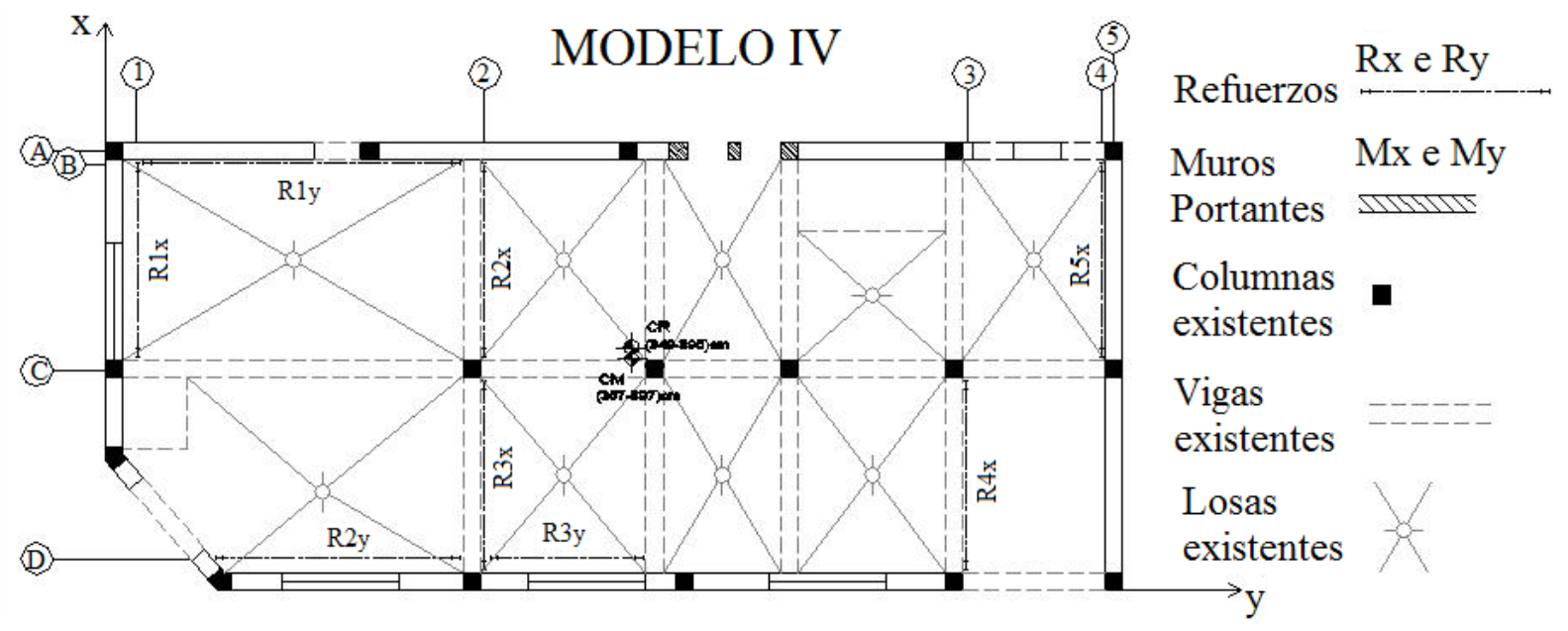

Figura 11. Planta estructura modelo de análisis IV

e) Envolvente de solicitaciones

De las solicitaciones obtenidas sobre cada modelo analizado, se determina una envolvente a ser utilizada para el diseño de los refuerzos estructurales. Tabla 1 
Revista ALCONPAT, Volumen 1, Número 1, Enero-Abril 2011, Páginas 1 - 15

Tabla 1. Solicitaciones en cada modelo y envolvente

\begin{tabular}{|c|c|c|c|c|c|}
\hline Elemento & Modelo I & Modelo II & Modelo III & Modelo IV & Envolvente \\
\hline $\mathrm{R} 1 \mathrm{x}$ & $134 \mathrm{kN}$ & $133 \mathrm{kN}$ & $135 \mathrm{kN}$ & $136 \mathrm{kN}$ & $136 \mathrm{kN}$ \\
\hline $\mathbf{R} 2 \mathbf{x}$ & $80 \mathrm{kN}$ & $85 \mathrm{kN}$ & $81 \mathrm{kN}$ & $86 \mathrm{kN}$ & $86 \mathrm{kN}$ \\
\hline $\mathbf{R 3 x}$ & $80 \mathrm{kN}$ & $85 \mathrm{kN}$ & $81 \mathrm{kN}$ & $86 \mathrm{kN}$ & $86 \mathrm{kN}$ \\
\hline $\mathbf{R} 4 \mathbf{x}$ & $72 \mathrm{kN}$ & $117 \mathrm{kN}$ & $72 \mathrm{kN}$ & $112 \mathrm{kN}$ & $117 \mathrm{kN}$ \\
\hline R5x & $67 \mathrm{kN}$ & $116 \mathrm{kN}$ & $67 \mathrm{kN}$ & $122 \mathrm{kN}$ & $122 \mathrm{kN}$ \\
\hline M1x & $109 \mathrm{kN}$ & - & $109 \mathrm{kN}$ & - & - \\
\hline R1y & $152 \mathrm{kN}$ & $152 \mathrm{kN}$ & $229 \mathrm{kN}$ & $230 \mathrm{kN}$ & $230 \mathrm{kN}$ \\
\hline $\mathbf{R} 2 \mathbf{y}$ & $112 \mathrm{kN}$ & $119 \mathrm{kN}$ & $145 \mathrm{kN}$ & $147 \mathrm{kN}$ & $147 \mathrm{kN}$ \\
\hline $\mathbf{R 3 y}$ & $49 \mathrm{kN}$ & $51 \mathrm{kN}$ & $63 \mathrm{kN}$ & $64 \mathrm{kN}$ & $64 \mathrm{kN}$ \\
\hline M1y & $134 \mathrm{kN}$ & $134 \mathrm{kN}$ & - & - & - \\
\hline
\end{tabular}

\subsection{Diseño del refuerzo estructural}

Para el dimensionamiento de cada uno de los refuerzos estructurales, se siguen los lineamientos establecidos en INPRES-CIRSOC 103 Parte IV (INPRES-CIRSOC, 2000) (adaptación de la AISC). Se establece como filosofía de diseño la de permitir la plastificación por corte del elemento de enlace del pórtico para lo cual la condición de diseño es la de adoptar un enlace caracterizado como "corto". Los esfuerzos de diseño en el enlace son los que provienen de la envolvente de solicitaciones. Cada uno de los otros elementos constituyentes del pórtico se diseñan de modo tal que su comportamiento esté limitado al rango elástico, para lo cual los esfuerzos de diseño provienen a partir de la sobre resistencia al corte del enlace dada por la variabilidad de las tensiones y por el endurecimiento por deformación.

a) Características de los materiales

- Perfil de acero laminado (Bajo normas ASTM A-36)

Tensión de fluencia: $\quad \mathrm{f}_{\mathrm{y}}=253 \mathrm{MPa}$

Tensión de rotura: $\quad \mathrm{f}_{\mathrm{u}}=380 \mathrm{MPa}$

Variabilidad de tensiones: $\quad \mathrm{R}_{\mathrm{y}}=1.5$

- Platinas, platabandas, etc. (Bajo normas IRAM-IAS U 500-503 F-24)

Tensión de fluencia: $\quad \mathrm{f}_{\mathrm{y}}=240 \mathrm{MPa}$

Tensión de rotura: $\quad \mathrm{f}_{\mathrm{u}}=370 \mathrm{MPa}$

- Acero para hormigón armado (ADN 420 - Conformado en Frío)

Tensión de fluencia: $\quad \mathrm{f}_{\mathrm{y}}=420 \mathrm{MPa}$

- Hormigón (Grupo H-I, Clase de resistencia H -13, de acuerdo a CIRSOC 201, 1982)

Resistencia característica: $\quad \mathrm{f}_{\mathrm{c}}{ }_{\mathrm{c}}=13 \mathrm{MPa}$

Cemento tipo:

puzolánico

Contenido mínimo:

$320 \mathrm{~kg} / \mathrm{m}^{3}$

Asentamiento A-2:

de 5 a $9.5 \mathrm{~cm}$

Relación máx. a/c:

0.50

Tamaño máx. agreg. grueso: $25 \mathrm{~mm}$

El hormigón se utiliza solo en las fundaciones de los refuerzos, las cuales han sido consideradas como del tipo pozo romano. Éstas estarán sujetas a eventuales esfuerzos de levantamiento (tiro) y 


\section{Revista ALCONPAT, Volumen 1, Número 1, Enero-Abril 2011, Páginas 1 - 15}

compresión originados por la carga horizontal. El aporte de capacidad al tiro está dado por el peso propio del pozo más el peso propio de una cierta cuña de suelo circundante a la fundación. La capacidad a compresión estará dada por el área de la base del pozo, dando origen a valores de tensión actuantes en el hormigón, para los cuales una clase de resistencia $\mathrm{H}-13$ resulta suficiente.

b) Esquema de refuerzos adoptados

Los esquemas de los refuerzos adoptados se presentan en las Figuras 12, 13 y 14.

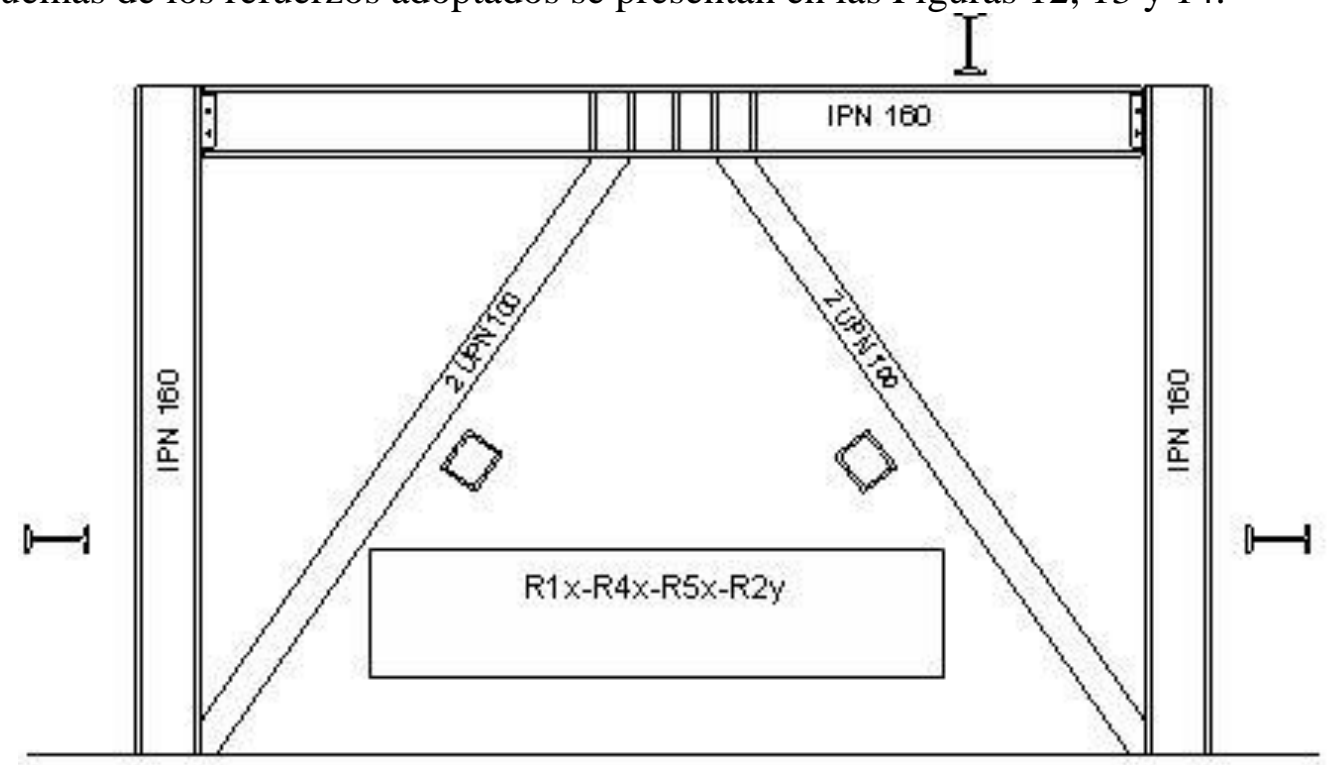

Figura 12. Refuerzo R1x-R4x-R5x-R2y

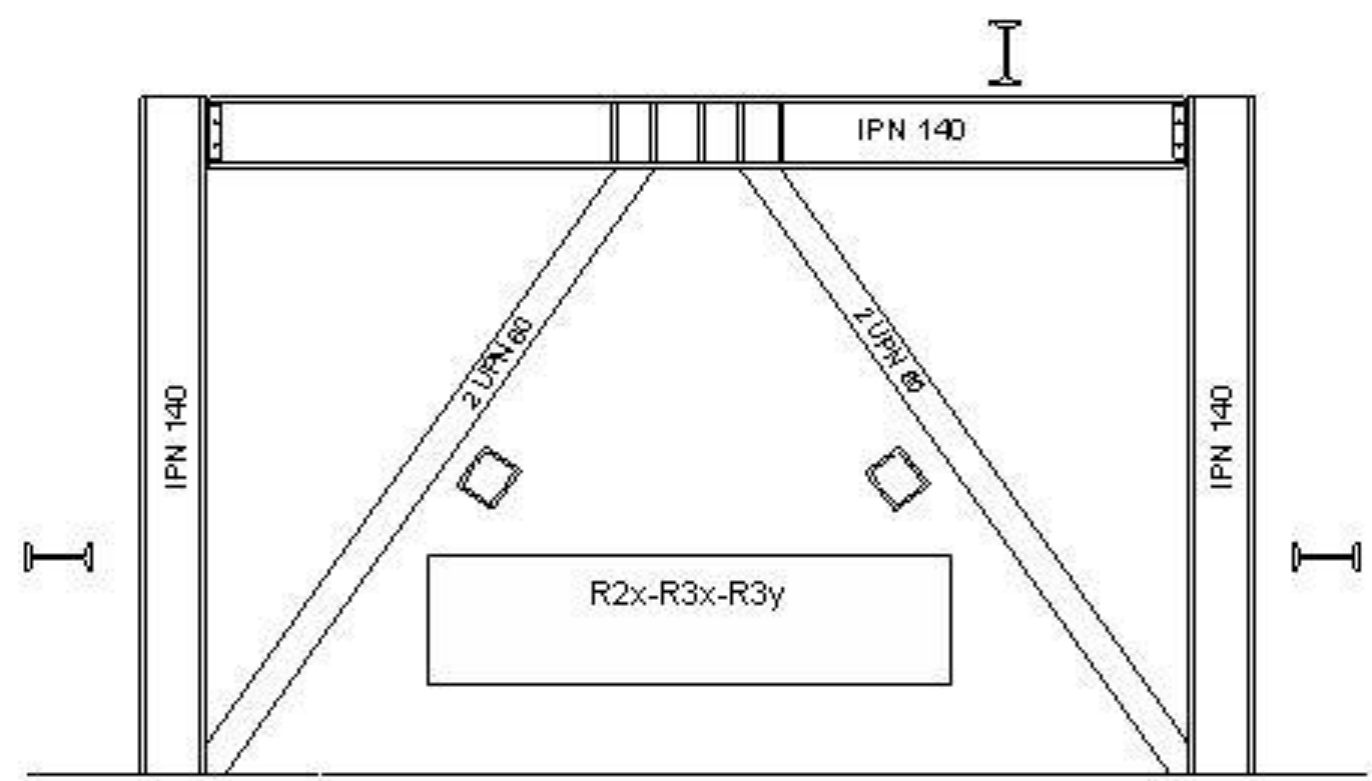

Figura 13. Refuerzo R2x-R3x-R3y 


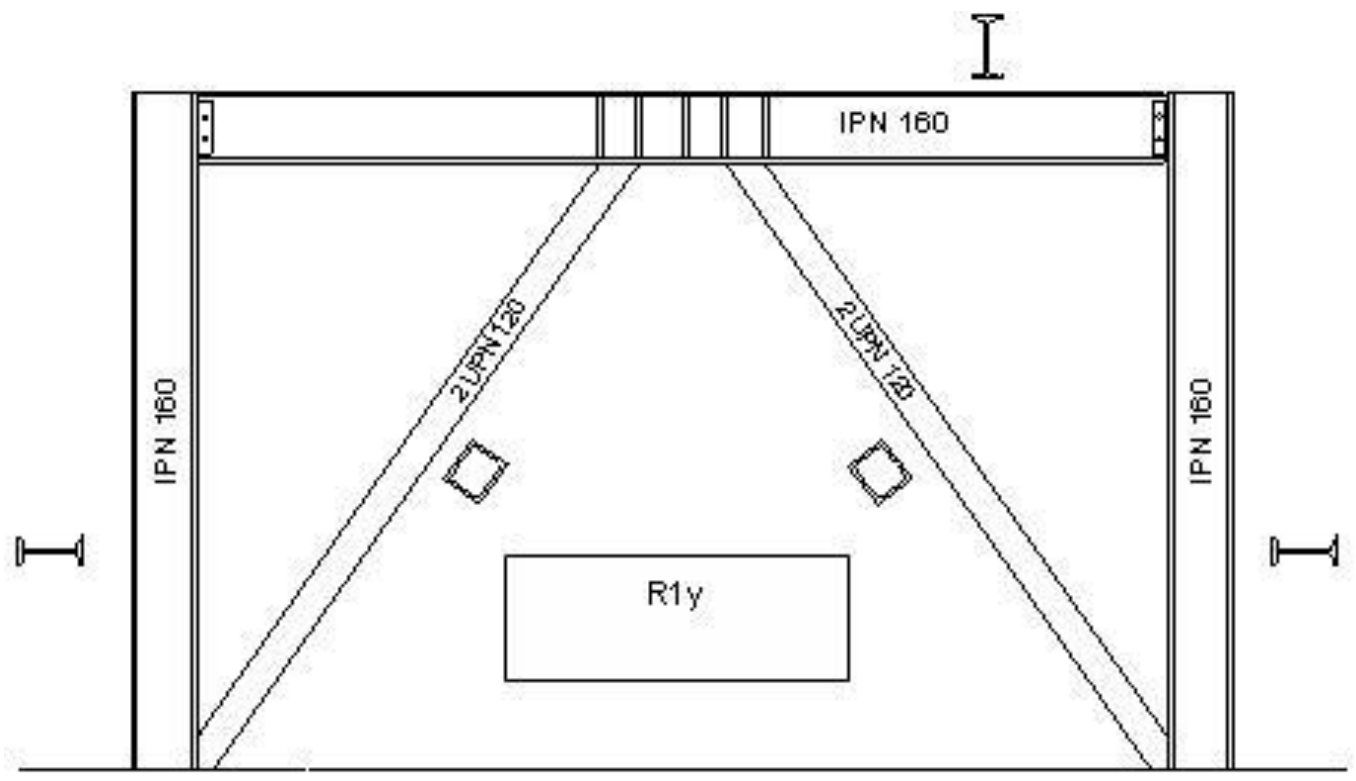

Figura 14. Refuerzo R1y

\subsection{Evaluación del desempeño del refuerzo}

Sobre cada uno de los refuerzos diseñados, se realiza un análisis estático no-lineal plano incremental (Pushover). Se presenta esquemáticamente la gráfica de la curva bilineal Corte Basal vs. Desplazamiento de la cima, correspondiente a los refuerzos. Figura 15.

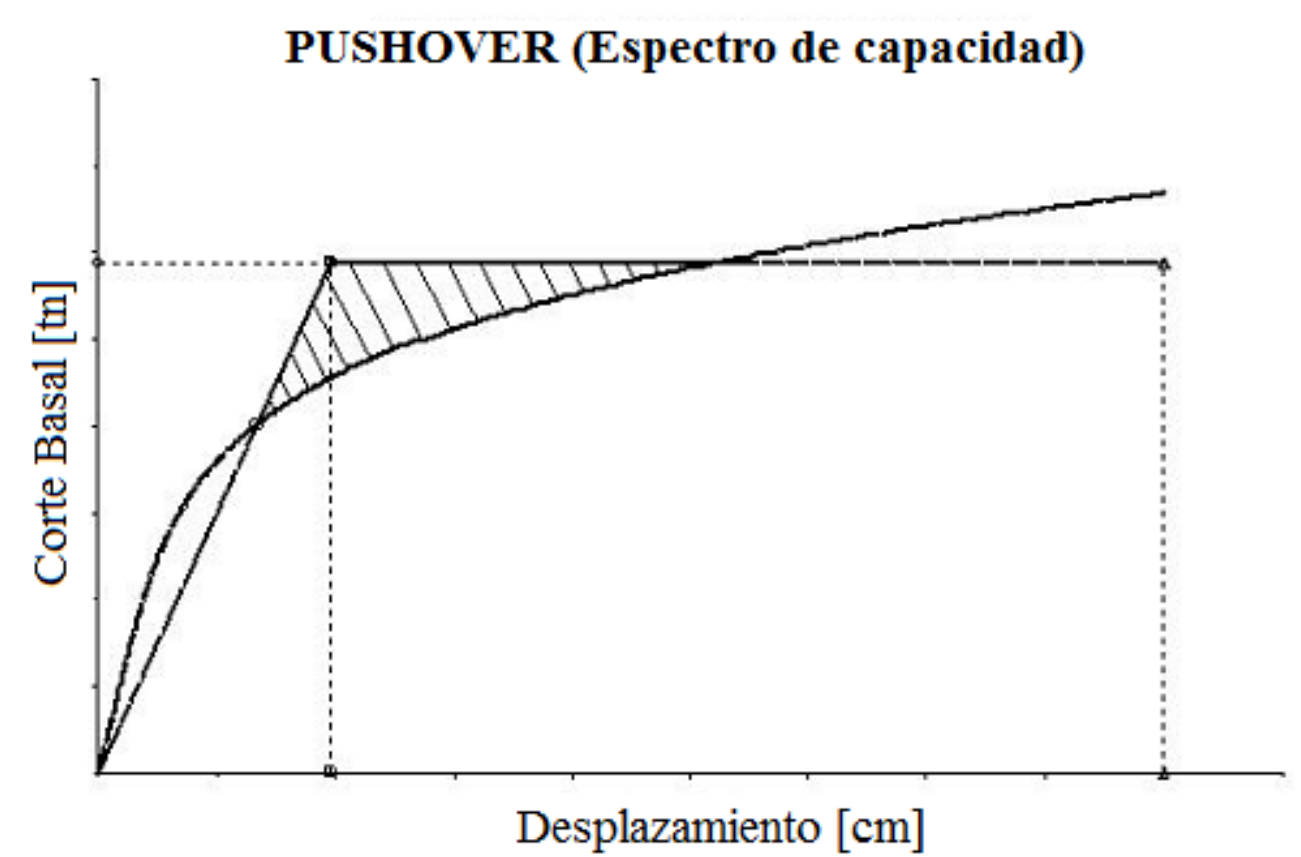

Figura 15. Curva Corte Basal vs. Desplazamiento de la cima

Luego con la capacidad de cada uno de los refuerzos se evalúa la capacidad global de la estructura mediante un análisis estático no-lineal incremental 3D. Resultó entonces ser la capacidad de la estructura en la dirección $\mathrm{x}-\mathrm{x}$ de: $\mathrm{Vu}=1370 \mathrm{kN} ; \mathrm{D}_{\mathrm{u}}=42 \mathrm{~mm} ; \mu_{\delta}=4.2$ y la distorsión de piso $\phi=1.40 \%$. Mientras que para la dirección $\mathrm{y}-\mathrm{y}: \mathrm{Vu}=1110 \mathrm{kN} ; \mathrm{D}_{\mathrm{u}}=45 \mathrm{~mm}$; $\mu_{\delta}=4.5$ y la distorsión de piso $\phi=1.50 \%$. Figura 16 
Revista ALCONPAT, Volumen 1, Número 1, Enero-Abril 2011, Páginas 1 - 15
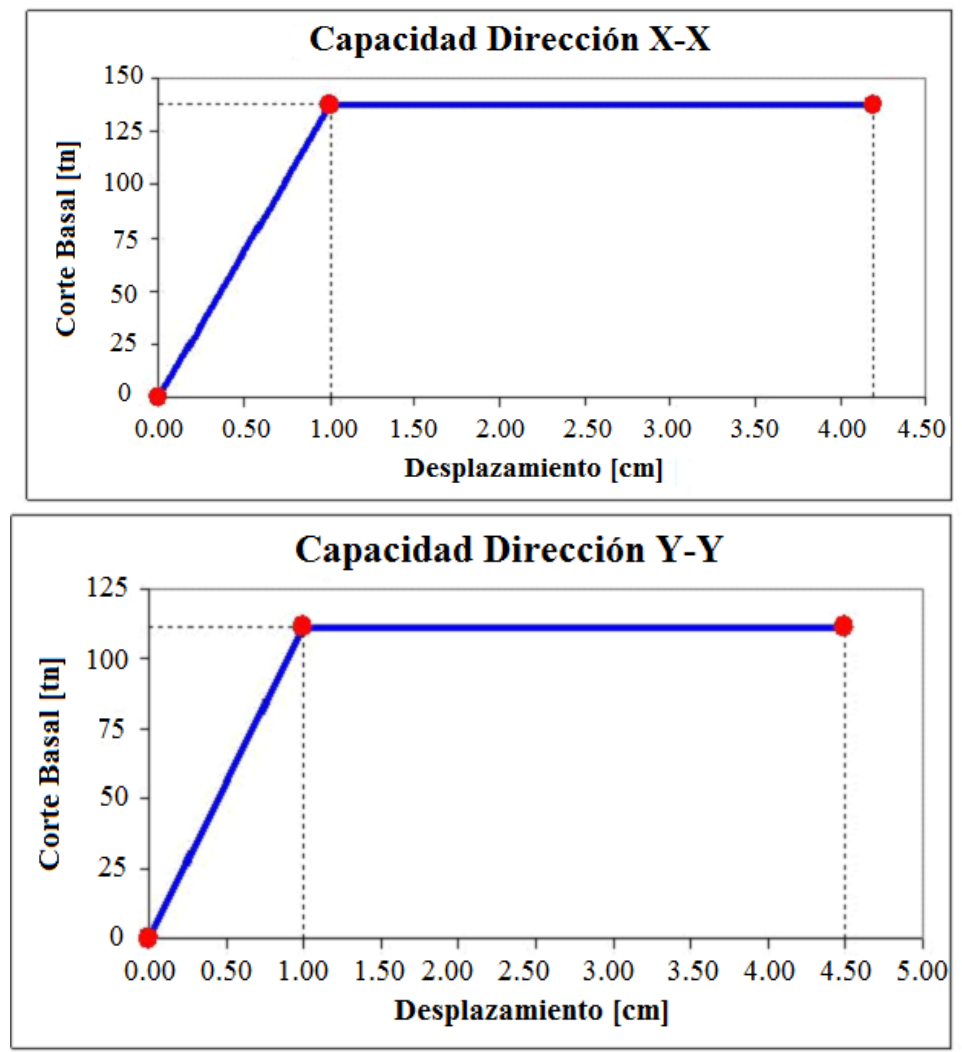

Figura 16. Capacidad Global de la estructura en cada dirección

a) Espectros de Diseño INPRES-CIRSOC 103 - Diagrama de Capacidad (Sae-Sd)

El objetivo del presente análisis es evaluar el desempeño del sistema de refuerzo frente a la demanda impuesta, adoptando como espectro de diseño el establecido en la propuesta del Proyecto de Reglamento Argentino para Construcciones Sismo Resistentes INPRES-CIRSOC 103 Parte I Construcciones en General, para una zona de elevado riesgo sísmico (zona 4) y para un suelo tipo 3. Figura 17.
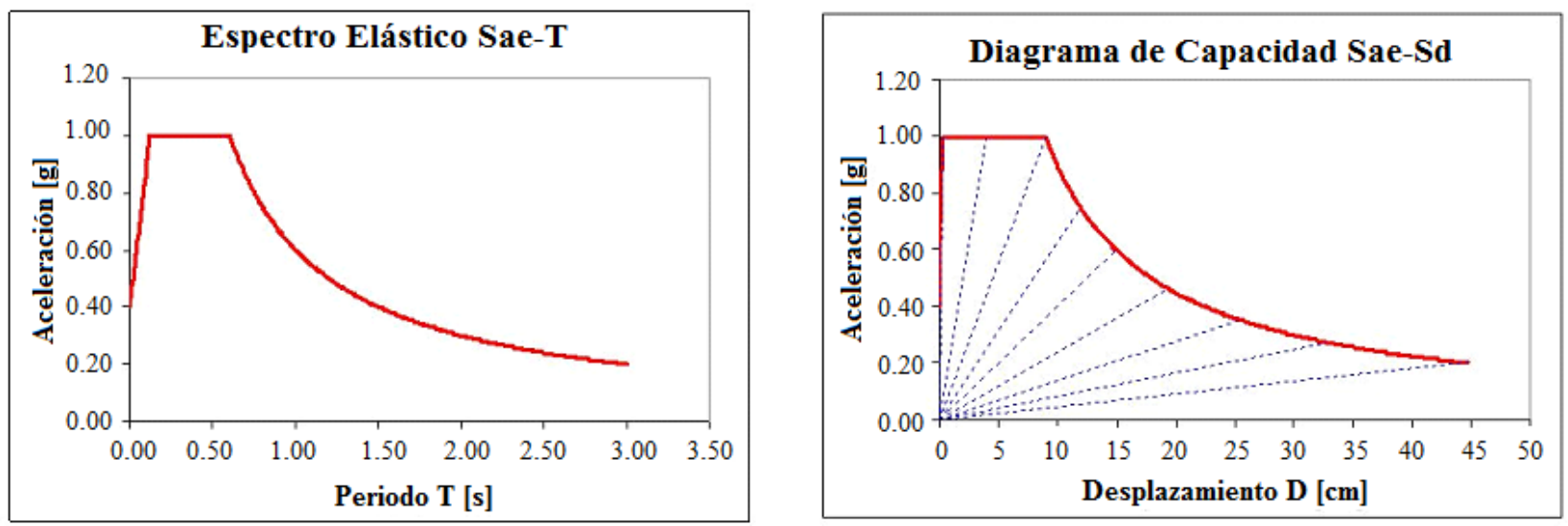

Figura 17. Espectro de Diseño - Diagrama de Capacidad

b) Evaluación de la demanda sobre la estructura en cada dirección

La demanda resultó ser en la dirección $\mathrm{x}-\mathrm{x}$ de: $\mathrm{S}_{\mathrm{d}}=15.4 \mathrm{~mm} ; \mu_{\delta}=1.54 \mathrm{y} \phi=0.51 \%$, mientras que para la dirección $\mathrm{y}-\mathrm{y}: \mathrm{S}_{\mathrm{d}}=21.9 \mathrm{~mm} ; \mu_{\delta}=2.19$ y $\phi=0.73 \%$. Figura 18 y Figura 19 
Revista ALCONPAT, Volumen 1, Número 1, Enero-Abril 2011, Páginas 1 - 15

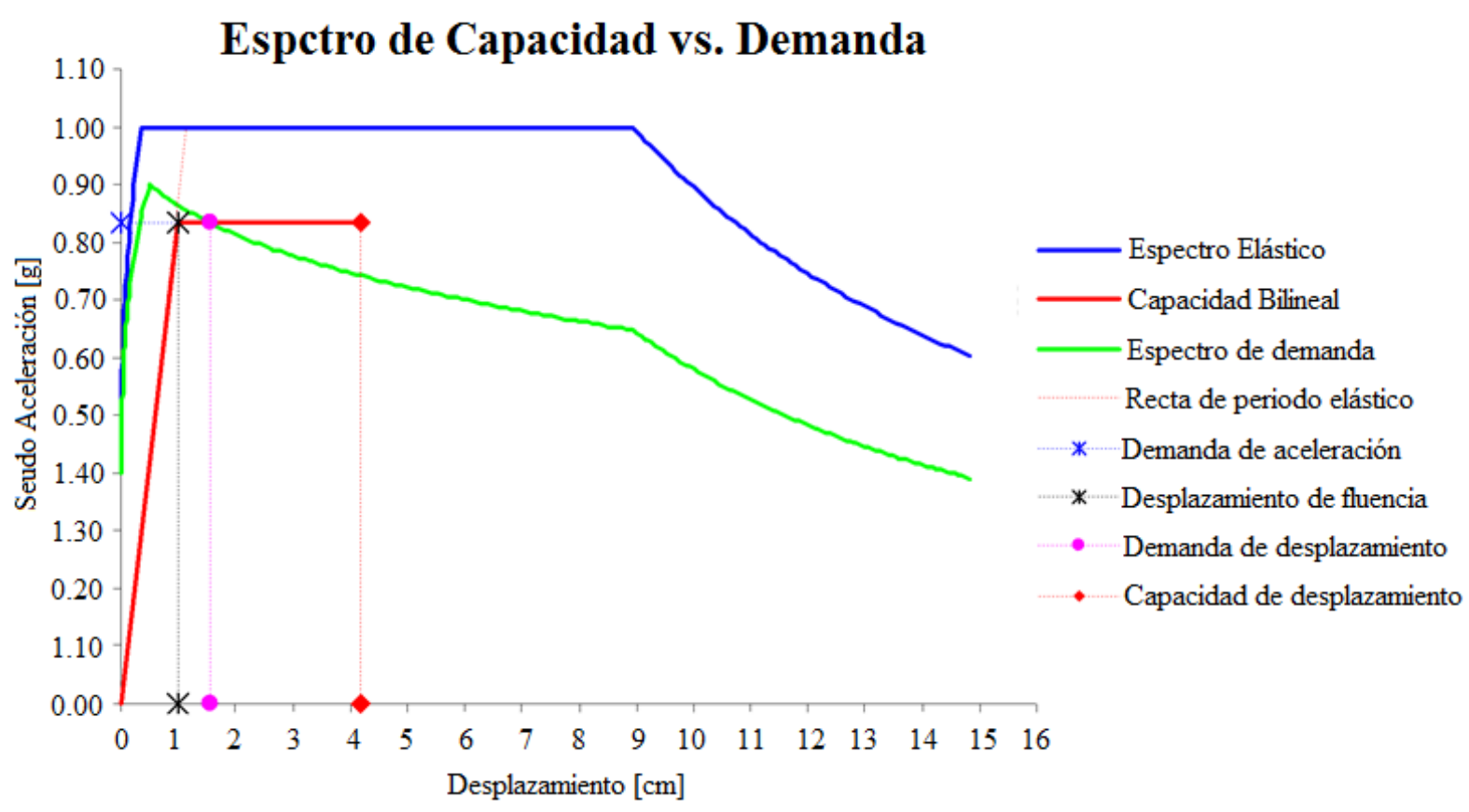

Figura 18. Demanda sobre la estructura en la dirección X-X

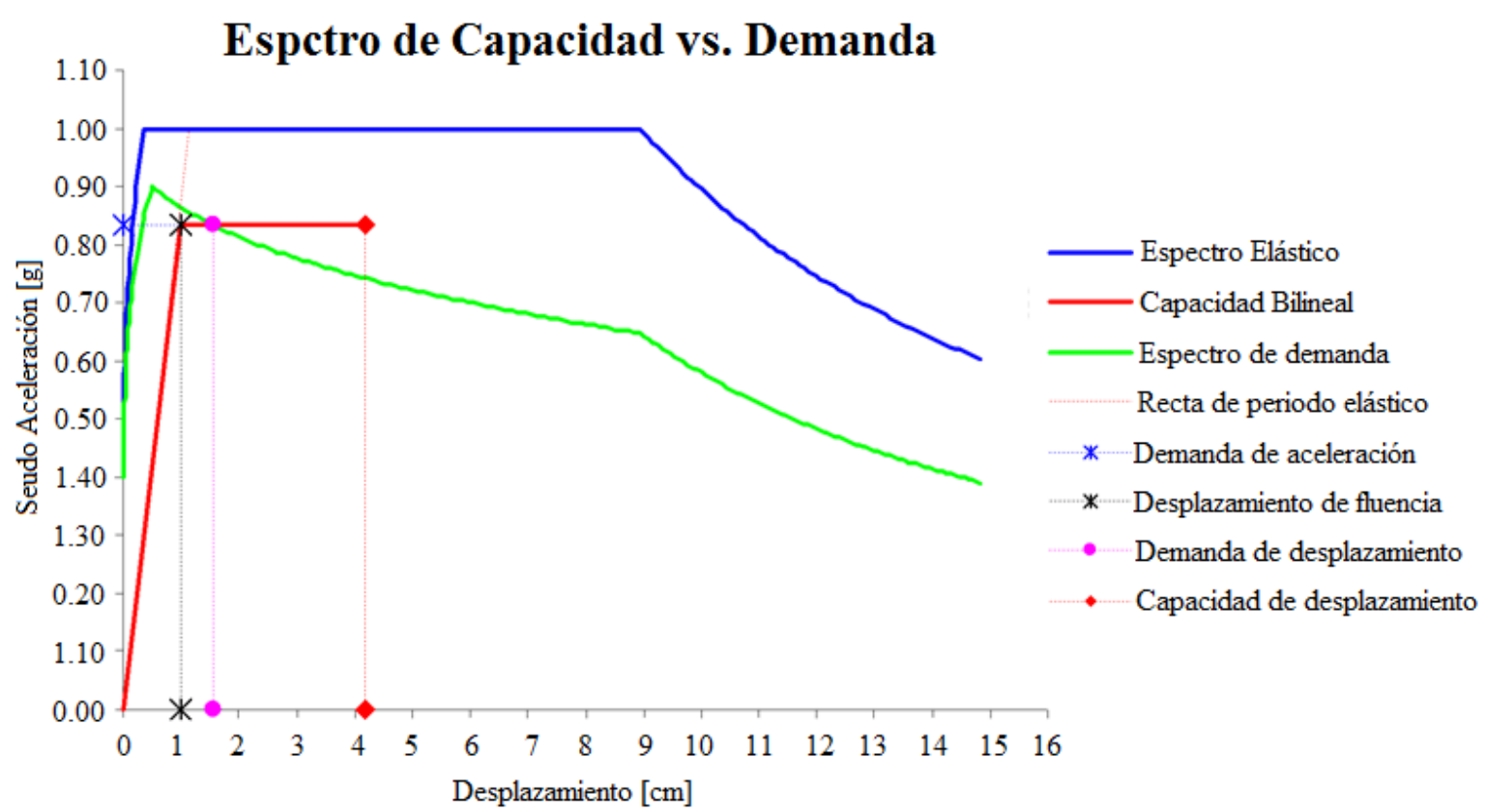

Figura 19. Demanda sobre la estructura en la dirección Y-Y

Adoptando como referencia lo propuesto por el comité VISION 2000 (Committee VISION 2000, 1995), el nivel de desempeño esperado se considera entre Operacional y de Seguridad. Tabla 2 
Revista ALCONPAT, Volumen 1, Número 1, Enero-Abril 2011, Páginas 1 - 15

Tabla 2: Niveles de desempeño vs. Distorsión de piso (Committee VISION 2000, 1995)

\begin{tabular}{|l|c|}
\hline Nivel de desempeño & Distorsión de piso $\phi[\%]$ \\
\hline Totalmente operacional & 0.20 \\
\hline Operacional & 0.50 \\
\hline Seguridad & 1.50 \\
\hline Próximo al colapso & 2.50 \\
\hline Colapso & $>2.50$ \\
\hline
\end{tabular}

\section{CONCLUSIONES}

\subsection{Conclusiones generales}

La metodología propuesta permite determinar la capacidad global de la estructura no sólo en términos de resistencia sino que también en términos de desplazamientos, pudiéndose evaluar la ductilidad de la estructura en cada una de las direcciones de análisis.

Se establecieron como criterios para la determinación de la capacidad individual de cada refuerzo, el de la generación de un mecanismo cinemático de rotulación conducente al colapso, y el del máximo desplazamiento admitido para la cima (1.5\% de la altura de piso, distorsión de piso). En todos los casos el último criterio fue el determinante, demostrando esto la necesidad de evaluar los desplazamientos dado que ignorarlos puede conllevar a daños importantes e inclusive al colapso estructural.

Del análisis de la capacidad global de la estructura en cada dirección, se observa que la distorsión de piso es similar (en $\mathrm{x}-\mathrm{x} \quad 1.40 \%$ y en y-y $1.50 \%$ ) a la de los refuerzos individuales, lo que indica que el diseño adoptado permite controlar adecuadamente los efectos torsionales.

A fin de poder llevar adelante el análisis de capacidad, cabe la necesidad de que los espectros de diseño elásticos a utilizar hayan sido originalmente preparados para que puedan pasar del formato seudo aceleraciones-periodo (Sae-T) al formato seudo aceleraciones-desplazamientos (Sae-Sd), y con ello obtener el diagrama o espectro de capacidad.

Del análisis de la demanda sobre la estructura en cada dirección, se observa un excelente comportamiento estructural del sistema adoptado, pudiéndose esperar para el terremoto de diseño un comportamiento considerado del tipo "operacional", pero pudiéndose también comportar como "segura" para un terremoto de mayor periodo de recurrencia que el de diseño.

\subsection{Conclusiones particulares}

Del análisis realizado para este caso de estudio, se permite concluir que el sistema de refuerzo propuesto ofrece una excelente capacidad de disipación de energía por deformación $\left(\gamma_{\mathrm{du}}=0.85\right)$ lo cual lo hace muy competitivo frente a otros sistemas de refuerzos estructurales. Además se encontró un adecuado control de los desplazamientos laterales $\left(S_{\mathrm{dmax}}=22 \mathrm{~mm}\right)$ permitiendo un muy buen desempeño de la estructura $\left(\phi_{\max }=0.73 \%\right)$.

El aporte de masa sísmica por parte de los refuerzos fue mínima, siendo del orden del $1.5 \%$ de la masa total de la estructura $(\mathrm{W}=24 \mathrm{kN})$, lo cual muestra su alta relación eficiencia/peso.

El análisis de costos (materiales y mano de obra) para la construcción de los refuerzos, permitió reflejar la poca incidencia que representa frente al costo total del inmueble (aproximadamente del $6 \%$ ).

Tanto la construcción de los refuerzos en taller, así como también la vinculación de éstos a la estructura a reforzar indicarían una rapidez considerable en la rehabilitación de la estructura, y con ello en su puesta en funcionamiento. 


\section{AGRADECIMIENTOS}

Se agradece el apoyo del CeReDeTeC, Facultad Regional Mendoza Universidad Tecnológica Nacional y Agencia Nacional de Promoción Científica y Tecnológica PICTO RS 255.

\section{REFERENCIAS}

AISC (2002), American Institute of Steel Construction, "Seismic Provisions for Structural Steel Buildings", USA.

Committee VISION 2000 (1995), SEAOC, Structural Engineers Association of California, USA. CIRSOC 201 (1982), Reglamento CIRSOC 201 y Anexos, Proyecto, Cálculo y Ejecución de Estructuras de Hormigón Armado y Pretensado, Argentina.

Consejo Profesional de Ingenieros, Agrimensores y Geólogos de Mendoza (1994), Código de Construcciones Sismo Resistentes Para la Provincia de Mendoza del año 1987, Mendoza, Argentina.

Gobierno de Mendoza (1970), Código de Construcciones Antisísmicas, Mendoza, Argentina. INPRES (1986), Publicación Técnica $N^{\circ}$ 10, “Gran Mendoza, el núcleo urbano expuesto al mayor nivel de riesgo sísmico en la República Argentina", Argentina.

INPRES - CIRSOC (2000), Proyecto de Reglamento Argentino para Construcciones Sismo Resistentes INPRES - CIRSOC 103 parte IV Construcciones de Acero, Argentina.

Popov, E.P. et al. (1989), Eccentrically Brace Frames: U. S. Practice", Engineering Journal, Vol. 26, No 2, (2 ${ }^{\text {da }}$ Qtr.), p. 66-80, AISC, Chicago, IL., USA. 